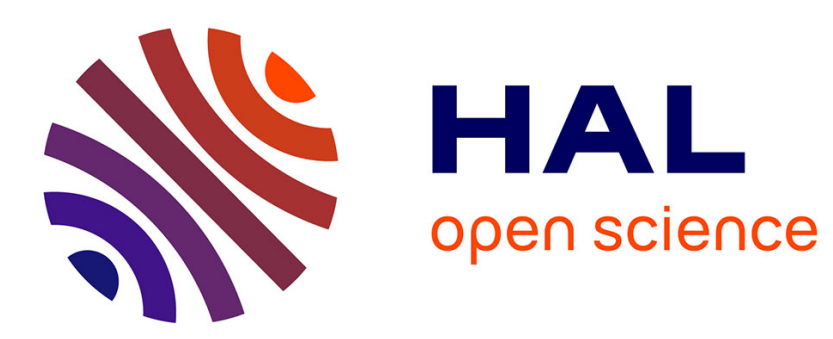

\title{
Extreme Statistics in Raman Fiber Amplifiers: From Analytical Description to Experiments
}

\author{
Kamal Hammani, Antonio Picozzi, Christophe Finot
}

\section{To cite this version:}

Kamal Hammani, Antonio Picozzi, Christophe Finot. Extreme Statistics in Raman Fiber Amplifiers: From Analytical Description to Experiments. Optics Communications, 2011, 284, pp.2594-2603. 10.1016/j.optcom.2011.01.057 . hal-00560484

\section{HAL Id: hal-00560484 https://hal.science/hal-00560484}

Submitted on 28 Jan 2011

HAL is a multi-disciplinary open access archive for the deposit and dissemination of scientific research documents, whether they are published or not. The documents may come from teaching and research institutions in France or abroad, or from public or private research centers.
L'archive ouverte pluridisciplinaire $\mathbf{H A L}$, est destinée au dépôt et à la diffusion de documents scientifiques de niveau recherche, publiés ou non, émanant des établissements d'enseignement et de recherche français ou étrangers, des laboratoires publics ou privés. 


\title{
Extreme Statistics in Raman Fiber Amplifiers: From
}

\section{Analytical Description to Experiments}

\author{
Kamal Hammani, Antonio Picozzi and Christophe Finot* \\ Laboratoire Interdisciplinaire Carnot de Bourgogne, UMR 5209 CNRS-Université de \\ Bourgogne, 9 avenue Alain Savary, BP 47870, 21078 Dijon, France \\ *Corresponding author: \\ E-mail address: christophe.finot@u-bourgogne.fr \\ Tel.: +33380395926
}

\begin{abstract}
:
In this work, we investigate the emergence of rare and intense events during the Raman fiber amplification of a continuous wave. We highlight how dispersive properties and pump depletion can strongly influence the statistical properties of the amplified signal and its optical spectrum. Under certain conditions, the probability density functions of the amplified signal are calculated analytically and compared with the results of the numerical simulations. The conclusions are qualitatively validated by experiments carried out at telecommunication wavelengths. (C) 2010 Elsevier
\end{abstract}

Keywords: Fiber optics; Raman amplifiers; extreme events; statistical optics 


\section{Introduction}

For more than three decades, optical fibers have been recognized as a versatile and highly effective testbed to investigate a very wide range of non-linear concepts, ranging from modulation instability [1] to the propagation of optical solitons [2] or self-similar pattern [3]. More recently, it has also been demonstrated that the supercontinuum generation of picosecond pulses or continuous waves in a highly nonlinear microstructured optical fiber can be characterized by an "extreme" statistics deviating strongly from usual Gaussian statistics : under the combined effects of spontaneous modulational instability and intrapulse Raman response, rogue solitons can emerge and be significantly shifted towards long wavelengths where they can then be adequately isolated by an adequate filtering [4-6]. Other studies have outlined that such statistics are not restricted to soliton-supporting systems and that the Raman effects is a key ingredient that promotes non-Gaussian statistics, either through cascaded Stokes generation [7], through a turbulent behaviour recorded in ultralong Raman fiber cavities [8] or through pulse-topulse collisions and energy exchanges as observed in telecommunication systems $[9,10]$._In this contribution, we focus on discrete Raman amplifiers where extreme statistics have also been numerically and experimentally highlighted [11-13]. As such amplifiers are key components of modern optical transmission technologies $[14,15]$, it is crucial to better understand the physical origin of such behaviour and to develop adequate means to characterize and predict the emergence of those rare but intense spikes of light.

It has already been shown that under the influence of a quasi-instantaneous gain and provided that the group velocity mismatch between the pump wave and the amplified Stokes (signal) wave can be neglected, the fluctuations of a partially incoherent pump can be 
exponentially transferred to the signal component. Similar conclusions have also been extended to the context of parametric amplification [16-18].

In the present article, we extend the work initiated in [11] and we further investigate the significant impact of two parameters of a discrete Raman fiber-based amplifier. On the one hand, we will show that the initial average signal power and the associated effect of pump depletion strongly influence the level of the extreme structures that can be detected. On the other hand, we will also stress the major impact of the pump-signal walk-off, i.e. the group velocity difference between the pump and signal waves. The consequences of both effects will be studied in the temporal and spectral domains.

Our paper will be thus organized as follows. We will first present the model we use as well as a simplified model that enables us to achieve some clear physical understanding of the underlying nonlinear physics. Then, we will more specifically investigate the amplification of a continuous wave in the Raman amplifier. Analytical expressions of the probability density functions of the signal intensity are calculated explicitly under certain conditions. Contrarily to the widely spread approach based on the relative intensity noise (RIN) derivation in the frequency domain [19-23], our results will be directly derived through an analysis in the temporal domain. Numerical simulations will confirm the trends obtained analytically. In order to provide an accurate and close to complete picture of the nonlinear dynamics, evolution of the optical spectrum will also be discussed. In the final part of the paper, we will describe a set of experiments that qualitatively validate our conclusions on the impact of the pump depletion and of the walk-off effect.

\section{Model}




\subsection{Description of the amplification process and approximations}

During its evolution in a single mode optical fiber, the slowly-varying envelope of the electric field $\psi(z, T)$ of an optical wave can be described by the extended nonlinear Schrödinger equation [24] :

$$
i \frac{\partial \psi}{\partial z}=-i \frac{\alpha}{2} \psi+\frac{\beta_{2}}{2} \frac{\partial^{2} \psi}{\partial T^{2}}+i \frac{\beta_{3}}{6} \frac{\partial^{3} \psi}{\partial T^{3}}-\gamma \psi \int_{0}^{\infty} R(T)|\psi(z, T-T)|^{2} d T^{\prime}
$$

with $\beta_{2}$ and $\beta_{3}$ describing the chromatic dispersive properties of the optical fiber, $\gamma$ the Kerr nonlinear coefficient (typically, we will investigate fibers with $\gamma=10 \mathrm{~W}^{-1} \cdot \mathrm{km}^{-1}$ ), $\alpha$ the optical losses, $R(t)$ the silica response function, which includes both the instantaneous Kerr contribution and the delayed Raman response function [24]. $z$ and $T$ are the propagation distance and the temporal coordinate in a reference frame moving at the group velocity of the wave. $\psi(0, T)$ is the initial field made of the temporal superposition of the pump wave $\psi_{P}$ and the signal $\psi_{S}$, characterized by the respective carrier frequencies $\omega_{P}$ and $\omega_{S}: \psi(0, T)=\psi_{P}(0, T)+\psi_{S}(0, T) \exp (-$ $i \Omega T$ ). The signal is shifted by $\Omega=\omega_{S}-\omega_{P}=-13 \mathrm{THz}$ with respect to the pump frequency and therefore leads to the maximum gain response .

As long as no additional frequency is generated by nonlinear frequency mixing or by cascading of the Raman process, it is possible to isolate the evolution of the pump $\psi_{P}$ from the evolution of the signal $\psi_{S}$. We can also consider that the Raman gain experienced by the signal is constant over the spectral range under investigation $g_{R}=6.5 \times 10^{-3} \mathrm{~m}^{-1}$ and that the Raman intrapulse effect and resulting soliton self-frequency shift are not significant in the various experiments carried out. Moreover, given the relatively short length of propagation considered in the experiment (typically half a kilometer), the consequences of the fiber linear losses can be 
neglected. Under these conditions, Eq. (1) can be reduced to the following set of two coupled nonlinear Schrödinger-like equations [14] :

$$
\left\{\begin{array}{l}
\frac{\partial \psi_{P}}{\partial z}=-\frac{\omega_{P}}{\omega_{S}} \frac{g_{R}}{2}\left|\psi_{S}\right|^{2} \psi_{P}-i \frac{\beta_{2 P}}{2} \frac{\partial^{2} \psi_{P}}{\partial T^{2}}+i \gamma\left[\left|\psi_{P}\right|^{2}+2\left|\psi_{S}\right|^{2}\right] \psi_{P} \\
\frac{\partial \psi_{S}}{\partial z}=\frac{g_{R}}{2}\left|\psi_{P}\right|^{2} \psi_{S}-i \frac{\beta_{2 S}}{2} \frac{\partial^{2} \psi_{S}}{\partial T^{2}}+i \gamma\left[\left|\psi_{S}\right|^{2}+2\left|\psi_{P}\right|^{2}\right] \psi_{S}+\delta \frac{\partial \psi_{S}}{\partial T}
\end{array}\right.
$$

with $\delta=1 / v_{g P}-1 / v_{g S}$ the walk-off parameter proportional to the difference between the group velocities $v_{g S}$ and $v_{g P}$ of the signal and the pump respectively. The ratio $\omega_{P} / \omega_{S}$ is very close to 1 so that we shall omit this term to simplify the analytical treatments. Furthermore, we have also found numerically that in the experimental conditions investigated here physical insight can be gained by neglecting the second order dispersion as well as the influence of self-phase modulation. Therefore, Eq. (2) can be further reduced to :

$$
\left\{\begin{array}{l}
\frac{\partial \psi_{P}}{\partial z}=-\frac{g_{R}}{2}\left|\psi_{S}\right|^{2} \psi_{P}+2 i \gamma\left|\psi_{S}\right|^{2} \psi_{P} \\
\frac{\partial \psi_{S}}{\partial z}=\frac{g_{R}}{2}\left|\psi_{P}\right|^{2} \psi_{S}+2 i \gamma\left|\psi_{P}\right|^{2} \psi_{S}+\delta \frac{\partial \psi_{S}}{\partial T}
\end{array}\right.
$$

This model is based on the evolution of the scalar electrical field, which then enables us to describe the pulse dynamics, both in the temporal and spectral domains. If we are only interested in the temporal intensity profile of the signal or pump waves $\left(P_{S}\right.$ and $P_{P}$ denoting the respective intensities, $P=|\psi|^{2}$ ), this model can be reduced to the well-known 'intensity model' usually employed to describe the Raman amplification process [14] :

$$
\left\{\begin{array}{l}
\frac{\partial P_{P}}{\partial z}=-g_{R} P_{P} P_{S} \\
\frac{\partial P_{S}}{\partial z}=g_{R} P_{P} P_{S}+\delta \frac{\partial P_{S}}{\partial T}
\end{array}\right.
$$


Let us note that our model has intentionally neglected the description of the spontaneous process of Raman amplification from quantum noise $[14,25,26]$ so that our conclusions solely result from the nonlinear transfer of pump to Stokes RIN.

\subsection{Initial signal and pump characteristics}

The initial signal will be considered as a purely continuous wave, i.e. a monochromatic wave, with an initial power $P_{S O}$. A crucial point is that the pump used in our study is a partially incoherent pump $\psi_{P O}$. To reproduce numerically the properties of this pump, we consider a random wave whose Fourier transform is Gaussian-shaped with $\delta$-correlated random spectral phases $\varphi(\omega)$ uniformly distributed between $-\pi$ and $\pi$ [27-31] :

$$
\tilde{\psi}_{P 0}(\omega) \propto \exp \left(-2 \ln (2) \frac{\omega^{2}}{\Omega_{L}^{2}}\right) \exp (i \varphi(\omega))
$$

$\Omega_{L}$ is the spectral full-width at half maximum (FWHM) of the pump, $\Omega_{L}=17.7 \mathrm{GHz}$, which corresponds in the temporal domain to large and ultrashort intensity fluctuations having a minimum temporal FWHM $T_{L}=0.44 / \Omega_{L}$ of 25 ps (Fig. 1(a) and associated inset). Note that, because the spectrum $\left|\widetilde{\psi}_{P 0}(\omega)\right|^{2}$ is $\delta$-correlated, the wave exhibits fluctuations that are statistically stationary in time [31]. More precisely, according to Eq.(5), the pump field $\psi_{P O}$ is characterized by a stationary Gaussian statistics, whose real and imaginary parts have the following probability density function pdf (we refer the reader to Ref. [31] for details):

$$
p d f(r, i)=\frac{1}{\pi\left\langle P_{P 0}\right\rangle} \exp \left(-\frac{r^{2}+i^{2}}{\left\langle P_{P 0}\right\rangle}\right)
$$


where $r=\operatorname{Real}\left(\psi_{\mathrm{P} 0}\right), i=\operatorname{Im}\left(\psi_{\mathrm{P} 0}\right)$ and with $\left\langle P_{P 0}>\right.$ being the average pump power (typically $\left\langle P_{P 0}>\right.$ $\cong 175 \mathrm{~mW})$.

Considering the standard pdf transformation rules [31], one obtains the following expression of the pdf of the pump power $P_{P O}$ [also see Fig. 1(b)] which is well-known for a polarized thermal source $[17,31]$ :

$$
p d f\left(P_{P 0}\right)=\frac{1}{\left\langle P_{P 0}\right\rangle} \exp \left(-\frac{P_{P 0}}{\left\langle P_{P 0}\right\rangle}\right)
$$

Note that the pdf of $P_{P 0}$ does not depend on $\Omega_{L}$ and that when plotted on a semilogarithmic scale, the respective plot exhibits a linear behaviour (Fig. 1(b)) [17]. The corresponding statistical distribution of the peak powers of the temporal structures of the pump is also of interest and is plotted on the inset of Fig. 1(b) (the statistics are based on the analysis of fluctuations over a period of $0.1 \mu \mathrm{s})$. We note that there is a non-negligible probability to observe a structure having a peak-power more than ten times the average power, as illustrated by Fig. 1(a) where the peak may reach an intensity 24 times larger than the average value. Compared to previous studies dealing with fluctuations of a few tens of percents of the peak-power of pulses delivered by nanosecond Q-switched laser pumps [13, 32-34], the statistically Gaussian partially incoherent pump considered here exhibits much larger deviations. 

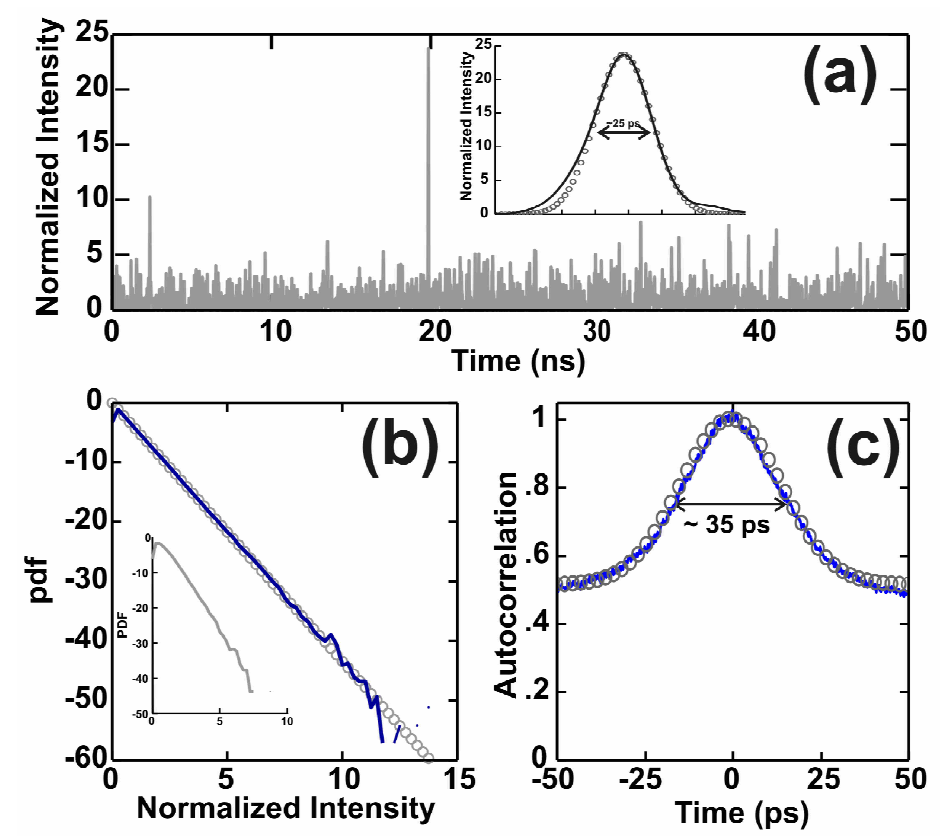

Fig. 1 : Pump properties : (a) Intensity fluctuations of the pump over a long duration (50 ns). Details of a peak are shown in the inset and are compared with a Gaussian fit (grey circles). Results are normalized by the average pump power $\left\langle\mathrm{P}_{\mathrm{P} 0}\right\rangle$. (b) Pdf of the pump intensity plotted on a logarithmic scale. Results of numerical evaluation of the pdf are compared with Eq. 7 (circles). The inset shows the distribution of the peak powers of the structures. Results are normalized by $\left\langle\mathrm{P}_{\mathrm{P} 0}\right\rangle$ (c) Intensity autocorrelation $\Gamma$ of the pump : experimental results (solid line) are compared with the model based on Eq. 5.

The pdf of the pump and signal can be experimentally recorded [17, 35], but this technique unfortunately requires onerous dedicated devices. An indirect but more convenient mean to have an idea of the pump and signal temporal fluctuations is the normalized measure of the intensity autocorrelation function $\Gamma$, whose general definition reads [31] :

$$
\Gamma(\tau) \triangleq\langle P(t+\tau) P(t)\rangle=\frac{\lim _{T \rightarrow \infty} \frac{1}{T} \int_{-T / 2}^{T / 2} P(t+\tau) P(t) d t}{\lim _{T \rightarrow \infty} \frac{1}{T} \int_{-T / 2}^{T / 2} P^{2}(t) d t}
$$

The autocorrelation function is characterized by two quantities: the temporal width of the autocorrelation $\Sigma$ and the contrast ratio $\Upsilon$ of the autocorrelation. $\Sigma$ is the full width at half maximum of the function $\Gamma(\tau)-\lim _{\tau \rightarrow \infty} \Gamma(\tau) . \Upsilon$ is the ratio of the maximum value of $\Gamma$ over its 
minimum value, i.e $\Upsilon=\Gamma(0) / \lim _{\tau \rightarrow \infty} \Gamma(\tau)$. We recall that, for a field characterized by Gaussian statistics (see Eq. 5), the variance of its power fluctuations equals two times its mean power, so that of $\Upsilon=2 \quad[27,31]$.

While the intensity autocorrelation function is convenient to understand some fluctuation properties of the intensity profile in the temporal domain, it does not provide any information on the temporal phase of individual fluctuations or on the spectral evolution of the optical waves. In this work, we will also focus our attention on the optical spectrum $S(\omega)$ defined as :

$$
S(\omega)=\left|\int_{-\infty}^{\infty} \psi(t) e^{i \omega T} d T\right|^{2}
$$

Note that, according to the Wiener-Khinchin theorem, the optical spectrum of a random wave characterized by a stationary statistics is the Fourier transform of the autocorrelation function of the field amplitude, $\Gamma_{\Psi}(\tau)=<\Psi(t+\tau) \Psi^{*}(t)>[31]$.

\subsection{Simplified model for the pump}

In order to get a physical insight into the impact of the spikes of the pump wave on the Raman amplification process, let us discuss a qualitative approach based on a simplified representation of pump fluctuations. As can be seen in the inset of Fig. 1(a), the most intense fluctuations of the pump can be reasonably fitted by a single Gaussian pulse with a FWHM temporal width $T_{L}$ of 25 ps corresponding to the shortest temporal width possible in the incoherent pump structure. Consequently, we will get interested in the amplification resulting from this single Gaussian structure. In other terms, the pump wave may be regarded, in a lose sense, as a collection of such 
individual Gaussian pulses. For such a temporal width and for the dispersion values involved in this study, the characteristic length $T_{0}^{2} / \beta_{2}$ (with $T_{0}$ the half width at $1 / e$ of the structure defined by $T_{0}=T_{L} /\left(2(\ln 2)^{1 / 2}\right)$ ) for which dispersion leads to significant impact has been found superior to $150 \mathrm{~km}$, which is several order of magnitude above our amplifier length $(500 \mathrm{~m})$ or above the typical walk-off length [26]. In other words, we confirm here our choice to neglect the influence of $\beta_{2}$ in our model. Based on the peak powers that have been recorded (inset Fig. 1b), we considered a peak-power of $2.5 \mathrm{~W}$ which typically corresponds to rare but very intense fluctuations.

\section{Influence of the initial signal average power}

\subsection{Depletion of the pump}

In this section, we investigate how the average power $P_{S O}$ of the initial continuous signal level can substantially influence the properties of the resulting intensity fluctuations as well as its spectral broadening. We neglect here the influence of the walk-off $(\delta=0)$, whose study will be the subject of the next section. Consequently, the evolutions of the pump and signal powers are described by the reduced equations (4):

$$
\left\{\begin{array}{l}
\frac{\partial P_{P}}{\partial z}=-g_{R} P_{P} P_{S} \\
\frac{\partial P_{S}}{\partial z}=g_{R} P_{P} P_{S}
\end{array}\right.
$$

This set of equation can be analytically solved to obtain the power profiles [25] : 


$$
\left\{\begin{array}{l}
P_{S}(z, T)=\frac{\left(P_{P 0}(T)+P_{S 0}\right) P_{S 0} e^{z g_{R}\left(P_{P 0}(T)+P_{S 0}\right)}}{P_{S 0} e^{z g_{R}\left(P_{P 0}(T)+P_{S 0}\right)}+P_{P 0}(T)} \\
P_{P}(z, T)=\frac{\left(P_{P 0}(T)+P_{S 0}\right) P_{P 0}(T)}{P_{P 0}(T)+P_{S 0} e^{z g_{R}\left(P_{P 0}(T)+P_{S 0}\right)}}
\end{array}\right.
$$

Assuming that the initial signal power is well below the pump power $\left(P_{S 0} \ll P_{P 0}\right)$, the following solution provides an accurate approximation of pump and signal evolutions :

$$
\left\{\begin{array}{l}
P_{S}(z, T)=\frac{P_{P 0}(T) P_{S 0}}{P_{S 0}+P_{P 0}(T) e^{-z g_{R} P_{P 0}(T)}} \\
P_{P}(z, T)=\frac{P_{P 0}^{2}(T)}{P_{P 0}(T)+P_{S 0} e^{z g_{R} P_{P 0}(T)}}
\end{array}\right.
$$

For the range of parameters considered in this paper, we compared this analytical solution with the results obtained by the numerical integration of the system (10).

\subsection{Simulations based on a simplified model}

\subsubsection{Temporal study}

Let us first investigate the consequences of pump depletion in the context of the simplified model discussed in Sec. 2C. We have plotted in Fig. 2(a) the initial pump and amplified signal for two initial average powers. As expected, the continuous seed has been amplified and a replica of the pump profile can be observed on the output signal. Due to the exponential gain, the temporal duration of the replica is lower than that of the pump. Depending on the initial average power of the seed, we can also note that the shape of the temporal profile is affected, the optical structure being longer in the case of higher initial power with a flattened top of the pulse. This effect is due to the nonlinear depletion of the pump wave. For higher signal powers, the gain 
experienced by the central part of the pulse saturates and the pump is highly depleted in the central region as confirmed by Fig. 2(b). Let us stress that for the range of power / fiber length considered here, it is not necessary to take into account the cascading of the Raman process: such a spontaneous cascading will ultimately lead to a depletion of the central part of the signal wave $[7,14,36,37]$.In these conditions, we can express the longitudinal evolution of the gain $G(z)$ that we define as the ratio of the peak power of the amplified signal and the initial average power (note that with such a definition, our gain differs from the ratio of the initial and output average powers).

$$
G(z)=\frac{\max \left(P_{S}(z, T)\right)}{P_{S 0}}
$$

This leads to the gain :

$$
\mathrm{G}(\mathrm{z})=\frac{P_{P}(z, T)}{P_{P_{0}}(T)} e^{z g_{R} P_{P 0}}
$$

If the depletion effects can be neglected, this expression reduces to well know small expression:

$$
\mathrm{G}(\mathrm{z})=\exp \left(g_{R} \max \left(P_{P 0}\right) z\right)
$$

Figure 2(c) reveals that even for $8 \mathrm{~mW}$ of initial average power, the gain is significantly reduced as compared to the results obtained with a seed of $0.2 \mathrm{~mW}$. We can also note that the impact of the pump depletion becomes more pronounced as the pump power increases. 

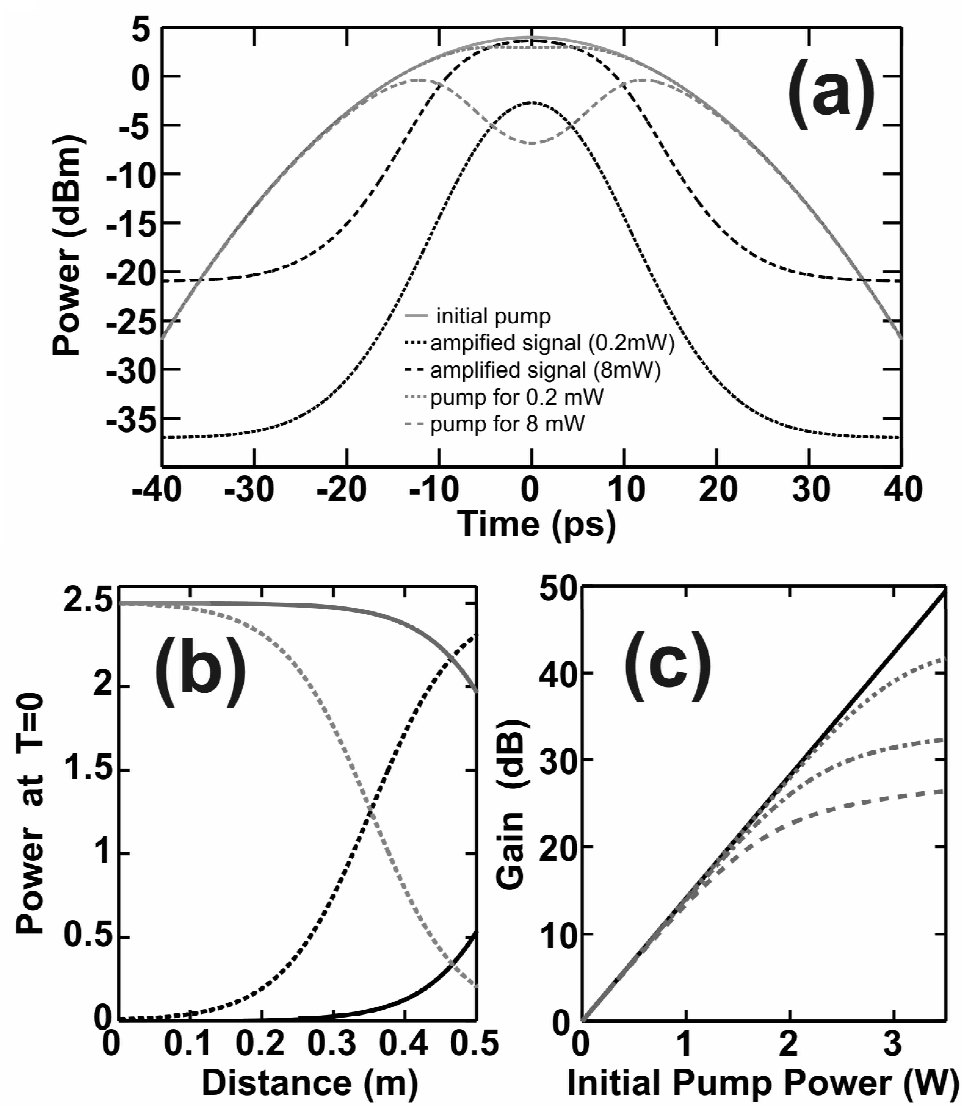

Fig. 2 : (a) Temporal intensity profiles. Amplified signal for an initial average power of $0.2 \mathrm{~mW}$ (black dotted line) and $8 \mathrm{~mW}$ (black dashed line). The initial pump (solid grey line) is compared to the pump after propagation for an initial signal power of $0.2 \mathrm{~mW}$ and $8 \mathrm{~mW}$ (grey dotted and grey dashed line respectively). Results are plotted on a semi-logarithmic scale (b) Longitudinal evolution of the pump and signal power at $T=O$ (grey and black lines respectively) for initial signal powers of $0.2 \mathrm{~mW}$ and $8 \mathrm{~mW}$ (solid and dotted line respectively). (c) Evolution of the gain versus the initial pump peak power for various initial average powers of the continuous seed $(0.2 \mathrm{~mW}, 2 \mathrm{~mW}$ en $8 \mathrm{~mW}$, dotted, mixed and dashed lines respectively). The solid black line shows the results in the absence of pump depletion, as predicted by Eq. 15 .

\subsubsection{Spectral study}

Let us now consider the spectral intensity profile of the amplified signal. As illustrated in Fig. 3(a), we note a significant spectral expansion of the initial continuous signal. The spectral broadening of the signal is essentially caused by the cross-phase modulation (XPM) of the pump on the signal, a feature that was already noticed in [38]. This is confirmed here in Fig. 3(a), 
which shows that the spectral broadening of the signal is considerably reduced in the absence of XPM effects [continuous line in Fig. 3(a)]. Note that such effect of XPM-induced spectral broadening may lead to a spectral selection of the rarest events [12] or may provide a convenient mean to reproduce the fluctuations of a strong signal of a weak seed [39].

From Eq. 3 and neglecting depletion effects, we can easily express the nonlinear phase shift resulting from XPM [24]:

$$
\phi_{X P M}(z, T)=2 \quad \gamma \quad P_{P 0}(T) z
$$

Taking the maximum of the time derivative of this expression, we can derive an estimate of the total spectral width $\Delta v$ of the amplified structure :

$$
\Delta v(z)=4 \sqrt{2} \gamma \max \left(P_{P_{0}}\right) \frac{e^{-1 / 2}}{T_{0}} z
$$

In this case, we can note that the spectral broadening does not depend on the initial signal level and is only linked to the initial pump peak power. If we now take into account the effect of pump depletion (i.e. the decrease of the pump power as well as the temporal pump depletion, as defined by Eq. 12), we need to solve the following equation:

$$
\frac{\partial \phi_{X P M}}{\partial z}=2 \gamma P_{P}(z, T)
$$

We solved this equation numerically and the resulting spectral broadening (as derived by taking the maximum of the time derivative of the phase) is plotted on Fig. 3(b). The observed trend is in reasonable agreement with the results obtained by the direct numerical integration of Eq. 3 (spectral broadening has been evaluated by the enlargement of the basis of the spectrum, 
taken at $-10 \mathrm{~dB}$ ). Note that an increase in the initial signal level leads to a drop of the spectral broadening of the output pulse. Indeed, as pump depletion gets significant, the amplitude of the pump drops quickly (see Fig. 2(b)), so that the effective length over which the pump XPM has an impact decreases dramatically.
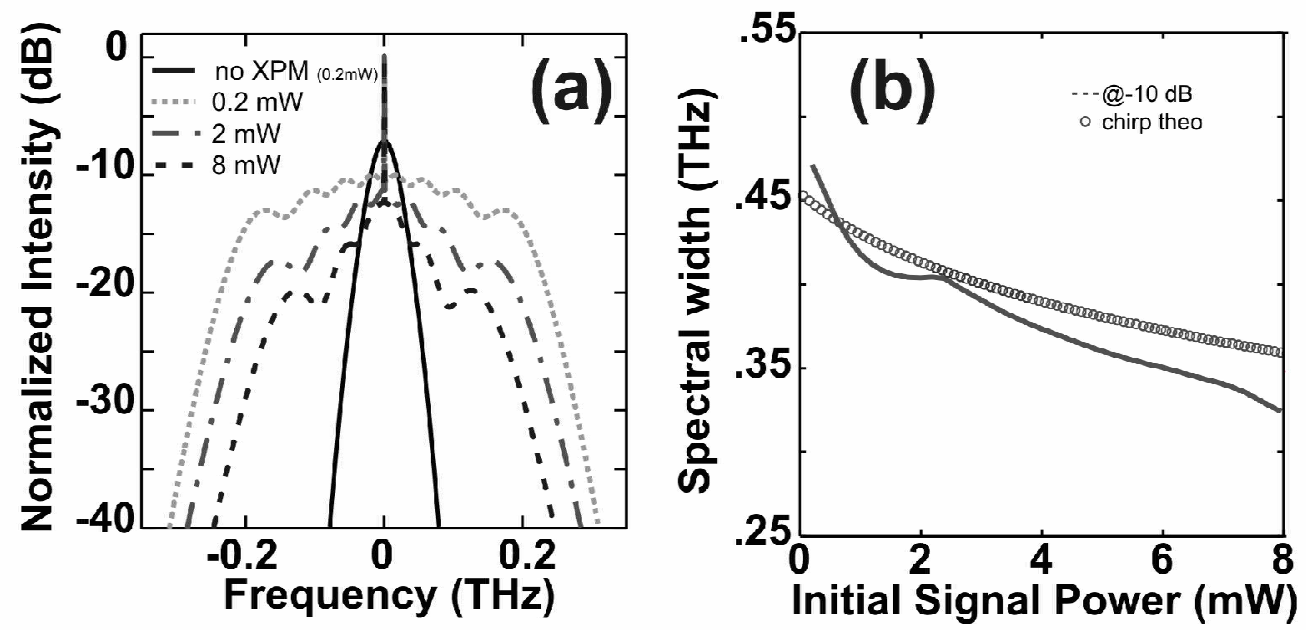

Fig. 3 : (a) Optical output spectrum of signal for three initial average powers : $0.2 \mathrm{~mW}, 2 \mathrm{~mW}$ and $8 \mathrm{~mW}$ (dotted light grey, mixed grey and dashed black lines respectively) compared to output spectrum of signal for $0.2 \mathrm{~mW}$ without XPM. (b) Evolution of the spectral width of the output structure as a function of the initial peak power : results obtained by the numerical resolution of system (3) (solid black line) are compared with the results derived from the numerical integration of Eq. 18 (grey line).

\subsection{Simulations based on an incoherent pump}

Let us now see whether the above trends obtained on the basis of the simplified model are reproduced by the complete model in which the signal is amplified by a genuine incoherent pump wave [see Eq. (3)]. From the pdf of the pump (Eq. 7) and the expression of the signal given in Eq. 12, we can express the pdf of the signal after amplification $[31,35]$ :

$$
p d f\left(P_{S}\right)=p d f\left(f^{-1}\left(P_{S}\right)\right) \cdot\left|\frac{d P_{P}}{d P_{S}}\right|
$$

where $f^{1}$ is the inverse of $f$ with $P_{s}=f\left(P_{p}\right)$. 
We remark that in the limit where pump depletion can be neglected, an expression of the pdf of the signal intensity can be derived in analytic form. Under the assumption that the pdf of the pump power is exponential (Eq. 7), one obtains

$$
p d f\left(P_{S}\right)=\frac{1}{g_{R} z P_{S}\left\langle P_{P 0}\right\rangle}\left(\frac{P_{S 0}}{P_{S}}\right)^{\frac{1}{g_{R} z\left\langle P_{P 0}\right\rangle}} H\left(P_{S}-P_{S 0}\right),
$$

where $H(x)$ denotes the Heaviside function. Equation (20) reveals an important property: the exponential pdf (7) of the pump power gives rise to an algebraic decay of the pdf (20) of the signal power. This algebraic long tail of the pdf reveals the existence of highly probable extreme events, which thus find their origin in the exponential and instantaneous nature of the Raman gain amplification. A remarkable consequence of such algebraic decay is the divergence of the average value of the signal power after only one amplification length of propagation, $\left\langle P_{S} / P_{S 0}\right\rangle(z)=\frac{1}{1-z /\left(g_{R}\left\langle P_{P}\right\rangle\right)}$. This divergence is saturated by the nonlinear regime of pump depletion, or by the mutual walk-off between the signal and pump waves (see Sec. 4B). Note that this divergence does not arise if the pump power were characterized by a Gaussian pdf, instead of the exponential pdf (7).

We have calculated numerically the pdf of the signal power given by Eq. 19 in the general case (including pump depletion) and the results are reported in Fig. 4(a). We have also compared the predictions of Eq. 20 with intensive numerical simulations. Even if advanced computational methods for rare events prediction are nowadays available [40], we have used in order get the numerical pdf a simple method based on the analysis of a long temporal signal. An excellent agreement between the numerical and analytical approaches has been found. More 
precisely, for a low amplification of the signal, the pdf obtained with and without depletion are similar and exhibit the algebraic long tail in agreement with Eq. 20, as illustrated in Fig. 4(a). The signal pdf in this linear regime of amplification strongly differs from the statistical properties of the pump [13, 32, 33, 41]. However, for higher initial signal levels, the impact of pump depletion can no longer be neglected and the value of the initial pump power becomes a fundamental upper limit. This merely explains why the signal tails are asymptotically limited by the exponential pdf of the pump (Eq. 7) : saturation of the gain strongly reduces the probability of the most extreme amplification factors [22] and the pdf ultimately exhibits a linear decrease in semilogarithmic scale (when no spontaneous cascading of the Raman process occurs during the amplification). This study also shows that the tails of the pdf distributions may be controlled in practice.
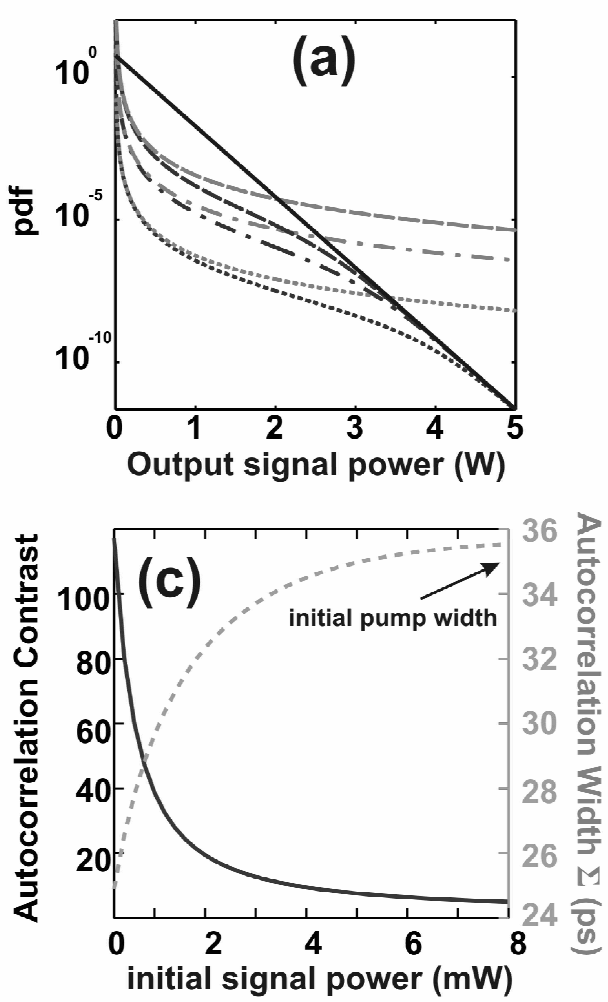
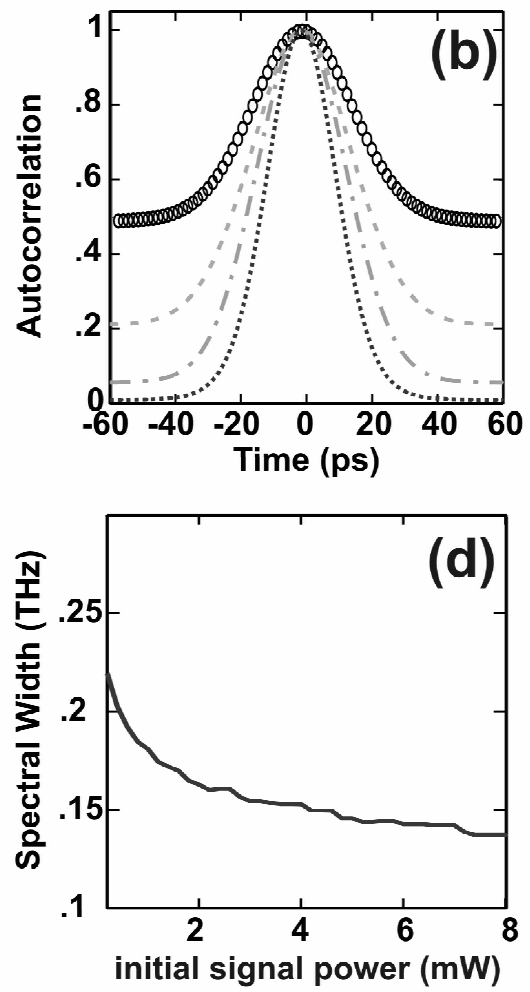
Fig. 4 : Influence of the initial average power (a) Pdf of the amplified signal for initial average signal power of $0.2 \mathrm{~mW}, 2 \mathrm{~mW}$ and $8 \mathrm{~mW}$ (dotted, mixed and dashed lines respectively), with (black) and without (grey) depletion. The results are compared with the pdf of the pump (solid black). (b) Evolution of the autocorrelation signal for various signal levels (same convention as subplot (a)) compared to the pump autocorrelation (black circles) (c) Evolution of the autocorrelation temporal width $\Sigma$ and of the autocorrelation contrast $\Upsilon$ according to the initial signal power. (d) Evolution of the spectral width of the optical spectrum.

Regarding the intensity autocorrelation, we can clearly see from Fig. 4(b) that the autocorrelation contrast $\Upsilon$ is far from 1 (i.e., the constrast of a continuous wave). On the contrary, the contrast is very high and is also clearly different from the contrast 2 of the Gaussian pump, suggesting different statistical properties for the signal and pump waves. Such an increase in the contrast $\Upsilon$ above the corresponding Gaussian value $(\Upsilon=2)$ simply reflects the existence of highly probable extreme events, as discussed above through the analysis of the pdf (20). The initial signal level also influences the autocorrelation shape as illustrated on Fig. 4(c) : one may note a change in the contrast - higher initial signal levels leading to lower contrasts. This corroborates the numerical results reported in Fig. 4(a), in which the saturation of the gain was shown to reduce the generation of extreme events. We can also remark that the temporal width of the autocorrelation signal broadens as the initial average powers are increased. Both effects are directly linked to the previously discussed consequences of pump depletion. Regarding the spectral evolution, we find that the signal is spectrally broadened by the incoherent pump amplification [38]. Similar trends discussed above on the basis of the simplified pump model are observed, i.e. a spectral broadening which is influenced by the initial signal level (Fig. 4(d)) with a decrease of the broadening for increasing level of initial signal.

\section{Impact of the dispersive properties of the fiber}

In this section, we shall focus our attention on the impact of the dispersive properties of the fiber and more specifically on the impact of the mutual walk-off $\delta$ between the signal and the pump 
waves. The effect of pump depletion is neglected here, so that our study is only relevant to relatively low signal intensities. It is a well-known fact that the walk-off $\delta$ tends to smooth the impact of the pump fluctuations on the amplified signal wave, simply because the walk-off averages out the pump fluctuations, i.e., it avoids a harmful pump to signal noise transfer [19]. Indeed, it has been shown that the maximum pump-signal velocity mismatch that is obtained in the counterpropagating pumping configuration was beneficial to lower the RIN of the amplified signal $[11,19]$.

\subsection{Simulations based on the simplified model}

Let us start by considering again the simplified model of the pump. Neglecting pump depletion effects allows us to reduce Eq. 3 into the single simplified equation:

$$
\frac{\partial \psi_{S}}{\partial z}=\frac{g_{R}}{2} P_{P 0} \psi_{S}+i 2 \gamma P_{P 0} \psi_{S}+\delta \frac{\partial \psi_{S}}{\partial T}
$$

whose solution reads

$$
\psi_{S}(z, T)=\psi_{S}(0, T) \exp \left(\frac{g_{R}}{2} \int_{0}^{z} P_{P 0}\left(0, T-\delta z^{\prime}\right) d z^{\prime}\right) \exp \left(2 i \gamma \int_{0}^{z} P_{P 0}\left(0, T-\delta z^{\prime}\right) d z^{\prime}\right)
$$

This leads to the following expression [24, 42] for the temporal intensity profile,

$$
P_{S}(z, T)=P_{S 0} \exp \left(g_{R} \frac{\sqrt{\pi} P_{P 0} T_{0}}{2 \delta}\left[\operatorname{erf}\left(\frac{T}{T_{0}}\right)-\operatorname{erf}\left(\frac{T-\delta z}{T_{0}}\right)\right]\right),
$$

and phase profile induced by XPM : 


$$
\phi_{X P M}(z, T)=2 \gamma \int_{0}^{z} P_{P 0}\left(0, T-\delta z^{\prime}\right)^{2} d z^{\prime}=\gamma \frac{\sqrt{\pi} P_{P} T_{0}}{\delta}\left[\operatorname{erf}\left(\frac{T}{T_{0}}\right)-\operatorname{erf}\left(\frac{T-\delta z}{T_{0}}\right)\right]
$$

The evolution of the output intensity profile of the signal due to the walk-off is summarized in Fig. 5(a). Increasing the value of the walk-off leads to a rapid drop of the peak intensity of the amplified structure, as well as a change in the temporal position of the maximum [12], the maximum of the signal at a distance $z$ being obtained for a temporal position $T_{C}=z \delta / 2$. The resulting gain, as defined by Eq. (13), and the FWHM temporal width of the optical structure are plotted in Fig. 5(b) and Fig. 5(c), respectively. We can clearly observe that, by increasing the walk-off, the gain decreases and the temporal width increases. Let us note that in the presence of pump depletion, the reduction of the gain becomes less apparent : neglecting pump depletion and for an integrated walk-off of $50 \mathrm{ps,} \mathrm{a} \mathrm{drop} \mathrm{of} \mathrm{the} \mathrm{gain} \mathrm{of} 17 \mathrm{~dB}$ is observed (Fig. 5b), which has to be compared to a drop of $7 \mathrm{~dB}$ in the presence of pump depletion (for a $8 \mathrm{~mW}$ initial signal). Indeed, walk-off is beneficial as it leads to an artificial regeneration of the pump seen by the signal $[26,43]$. Quite interestingly, we can also note that the temporal width is almost constant for a certain range of small values of the walk-off (typically for integrated walk-off below $25 \mathrm{ps}$ ). 

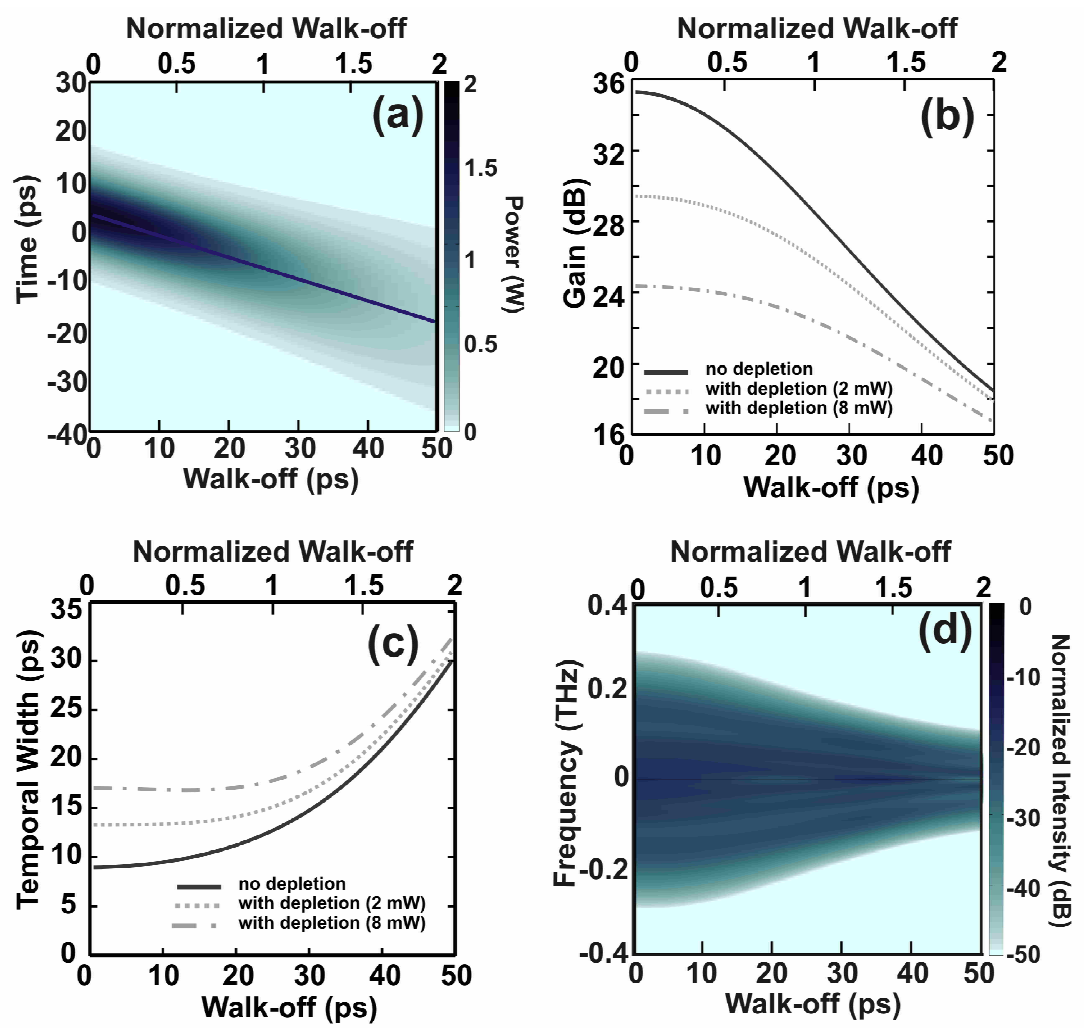

Fig. 5 : Influence of the integrated walk-off $\delta L$ in the simplified model. (a) Evolution of the temporal intensity profile. The maximum of the amplified structure is plotted with a dashed line. Evolution of the gain (b) and the temporal width (c) according to the integrated walk-off for the undepleted case (solid black line) and for the depleted case ( $2 \mathrm{~mW}$, dotted line and $8 \mathrm{~mW}$, mixed line). (d) Evolution of the spectral intensity profile of the output signal vs the integrated walk-off. A normalized scale is used to compare the integrated walk-off (ps) to the initial width of the pump $\left(T_{\mathrm{L}}=25 \mathrm{ps}\right)$.

The strong impact of the walk-off is also clearly visible in the spectral domain (see Fig. 5(d)). Indeed, higher values of $\delta L$ lead to a drop of the XPM induced by the pump on the signal. Such a decrease becomes critical when the integrated walk-off becomes of the same magnitude as the pump pulse duration [44]. Consequently, we observe a clear narrowing of the output signal spectrum as compared with the optical spectrum recorded without walk-off.

\subsection{Simulations based on an initial incoherent wave}


In order to check the validity of the qualitative conclusions obtained on the basis of our simplified model, we have run intensive numerical simulations of Eq. 3 with a partially incoherent pump wave (see Eq. 5). We compare in Fig. 6(a) the level of the fluctuations for four values of integrated walk-off $\delta$, i.e., 0 ps, 25 ps, 50 ps and 100 ps. As expected from our simplified model, we can see that the level of the peaks is influenced by the walk-off value, a higher integrated walk-off leading to a smoothing of the extreme events.

Under the assumption that the pdf of the pump power is exponential (Eq. 7), we have derived an analytical expression of the pdf of the signal intensity (see the Appendix):

$$
p d f\left(P_{S}\right)=\frac{1}{\left(\frac{z}{\varepsilon}-1\right) ! \quad\left(g_{R} \varepsilon\left\langle P_{P 0}\right)\right)^{\frac{z}{\varepsilon}}}\left(\frac{P_{S 0}}{P_{S}}\right)^{\frac{1}{g_{R} \varepsilon\left\langle P_{P 0}\right\rangle}} \frac{1}{P_{S}}\left[\ln \left(\frac{P_{S}}{P_{S 0}}\right)\right]^{\frac{z}{\varepsilon}-1} H\left(P_{S}-P_{S 0}\right)
$$

where $\varepsilon$ represents the effective correlation length of the pump as seen by the signal field due its walk-off with respect to the pump : $\varepsilon=\tau_{c} / \delta, \tau_{c}$ being the coherence time of the pump fluctuations that has been empirically found be close to $\sqrt{2} T_{L}$ (where $T_{\mathrm{L}}$ is defined p.6 after Eq.5). In the limit of a large effective correlation length $\varepsilon$ (i.e., negligible walk-off $\delta$, or large correlation time $\tau_{c}$ ), the expression of the pdf (25) recovers the pdf (20), in which walk-off effects were neglected $(\delta=0)$. Conversely, we show in the Appendix that in the limit of a small effective correlation length $\varepsilon$ (i.e., large $\delta$ or small $\tau_{c}$ ), the pdf (25) recovers the following expression $\operatorname{pdf}\left(P_{S}\right)=\delta\left[P_{S}-P_{S 0} \exp \left(g_{R}\left\langle P_{P}\right\rangle z\right)\right]$, where $\delta$ denotes here the Dirac $\delta$-function: as a result of the walk-off induced averaging mechanism, the signal grows as in if it were amplified by a fully coherent (deterministic) pump wave. 
In the same way it is also instructive to briefly comment the average value of the signal gain $\left\langle P_{S} / P_{S 0}\right\rangle$. Using the pdf (25) (or Eq. A2 in the Appendix), we obtain

$$
\left\langle\frac{P_{S}}{P_{S 0}}\right\rangle=\frac{1}{\left(1-g_{R}\left\langle P_{P}\right\rangle \mathcal{E}\right)^{z / \varepsilon}} .
$$

In the limit of a small effective correlation length $\varepsilon$ « 1 (large walk-off $\delta$ or small time correlation $\left.\tau_{c}\right)$, the average signal gain (26) recovers $\left\langle P_{S} / P_{S 0}\right\rangle=\exp \left(g_{R}\left\langle P_{P}\right\rangle z\right)$, as expected from the walk-off induced averaging of pump fluctuations. Conversely, in the opposite limit of a large effective correlation length $\varepsilon$ (i.e., negligible walk-off $\delta$, or large correlation time $\tau_{c}$ ), Eq. (26) recovers the expression of the average signal gain discussed in Sec. 3C, $\left\langle P_{S} / P_{S 0}\right\rangle=\frac{1}{1-z /\left(g_{R}\left\langle P_{P}\right\rangle\right)}$, which exhibits a divergence at one amplification length.

In Fig. 6(b) we compare the analytical expression of the pdf of the signal intensity (25) with the results of the numerical simulations. We remark that a satisfactory quantitative agreement is obtained between them. Figure 6(b) clearly shows that the influence of the walk-off is to reduce the long tails of the pdf of the signal intensity. We note that, while such a reduction is rather moderate for small values of the integrated walk-off ( $\delta L=0$ ps and $50 \mathrm{ps}$ ), the deviation becomes more pronounced as the walk-off parameter increases, with a significant drop of the probability of the most intense structures. The analytical prediction based on Eq. 25 has been found to be in good agreement with the results of the numerical simulations. 

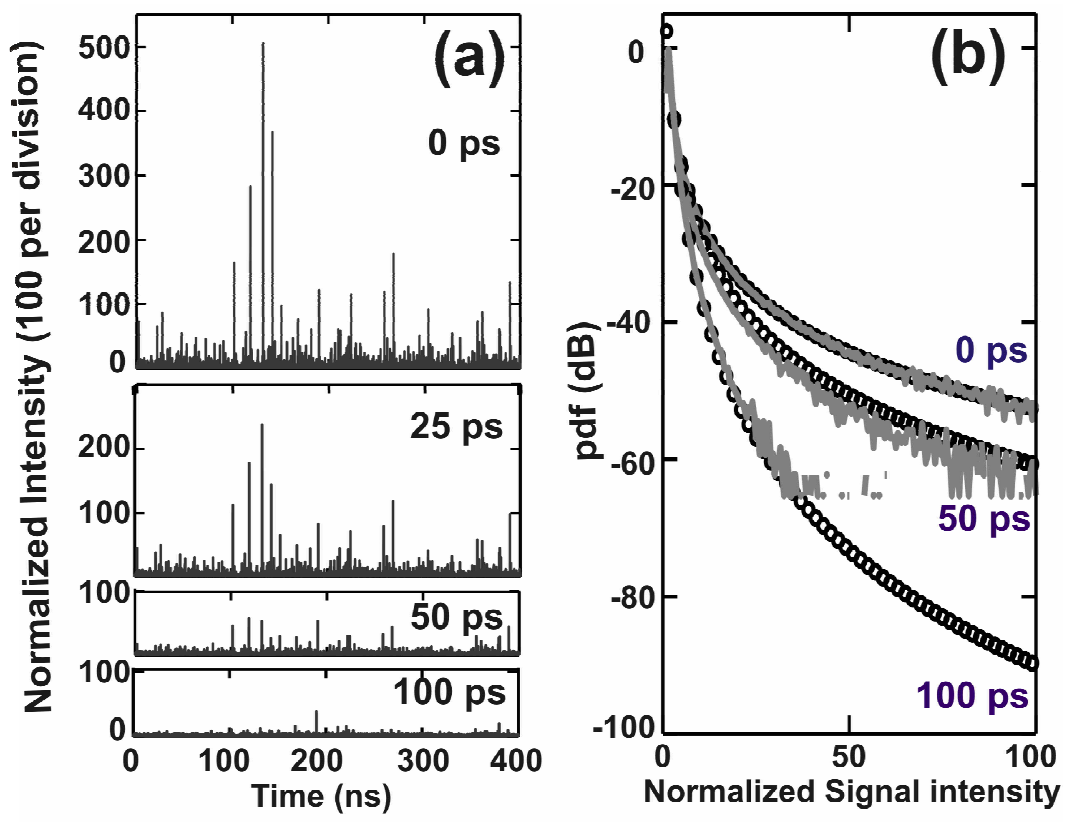

Fig. 6 : Impact of the walk-off in the complete model. (a) Temporal intensity evolution for four values of $\delta L: 0 \mathrm{ps}, 25 \mathrm{ps}, 50 \mathrm{ps}$ and $100 \mathrm{ps}$. (factor 100 per vertical division). (b) Corresponding pdf of the amplified signal (integrated walk-off values of $0 \mathrm{ps}, 50 \mathrm{ps}$ and $100 \mathrm{ps}$ which correspond to $0,2 T_{\mathrm{L}}$ and 4 $T_{\mathrm{L}}$ ). The analytical predictions of Eq. 25 (black circles) are compared with numerical simulations (grey solid lines).

The resulting intensity autocorrelation signals are plotted on Fig. 7(a). In agreement with the qualitative conclusions relying on the simplified model, we can note that the temporal width of the autocorrelation and the contrast (see Fig. 7(b)) are strongly influenced by the level of the integrated walk-off. More precisely, we can observe that for values ranging from $\delta L=0$ ps to $20 \mathrm{ps}$, the impact is moderate. Such a range of values has to be compared with the typical duration of the fluctuations (i.e., 25 ps at fwhm, 15 à 1/e). Above 20 ps, a rapid drop of the contrast combined with an increase of the temporal width can be observed. This is a consequence of the walk-off induced averaging mechanism discussed above through the pdf (25). As regard the contrast, the comparison between the curves obtained with low and high initial levels reveals that the same trends are observed, even in the presence of strong pump depletion, the resulting contrast being however lower and the temporal structures longer. 

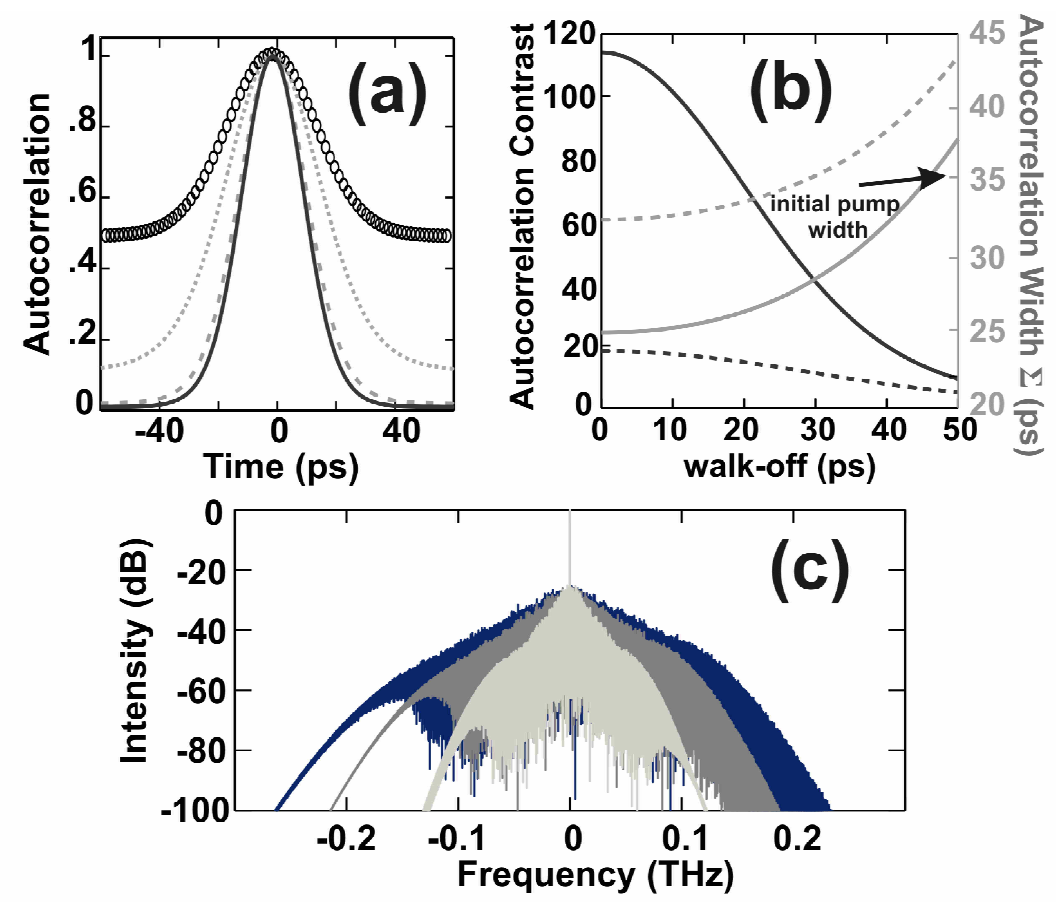

Fig. 7 : Impact of the walk-off in the complete model when neglecting pump depletion. (a) Intensity autocorrelation signal for integrated walk off values of $0 \mathrm{ps}, 25 \mathrm{ps}$ and $50 \mathrm{ps}$ (black solid, grey dashed and light grey dotted lines respectively) compared to the pump autocorrelation (black circles). (b) Evolution of the autocorrelation contrast (right, black solid line) and temporal width (left, grey dashed line) vs the integrated walk-off. Results obtained with an initial seed of $8 \mathrm{~mW}$ and taking into account pump depletion are plotted with a dashed line. (c) Output spectrum of the amplified signal for different values of $\delta L$ (same convention as subplot a).

\section{Experimental results}

\subsection{Experimental setup}

In order to validate our analytical and numerical predictions, we have used the experimental setup sketched in Fig. 8, which consists of an all-fibered set-up relying exclusively on commercially available telecommunication devices. The initial partially incoherent wave is delivered by a Raman laser with an output average power of $350 \mathrm{~mW}$, leading to an integrated average gain of

2. Given the unpolarized nature of this pump, we have taken into account a factor 2 when comparing the experimental results with the numerical simulations. The initial continuous signal 
is delivered by an external cavity laser. It is a polarized wave whose power can be adjusted between 0 and $10 \mathrm{~mW}$. A WDM coupler is used to combine the signal and the pump waves in a copropagative Raman scheme. The amplifier is based on a commercially available highly nonlinear fiber (ofs fibers) with dispersion flattened profiles. Several fibers with normal or anomalous dispersion have been tested, which has enabled us to test several values of the integrated walk-off. At the output, an optical autocorrelator and an optical spectrum analyser allowed us to measure the intensity autocorrelation function and the optical spectrum of the amplified signal.

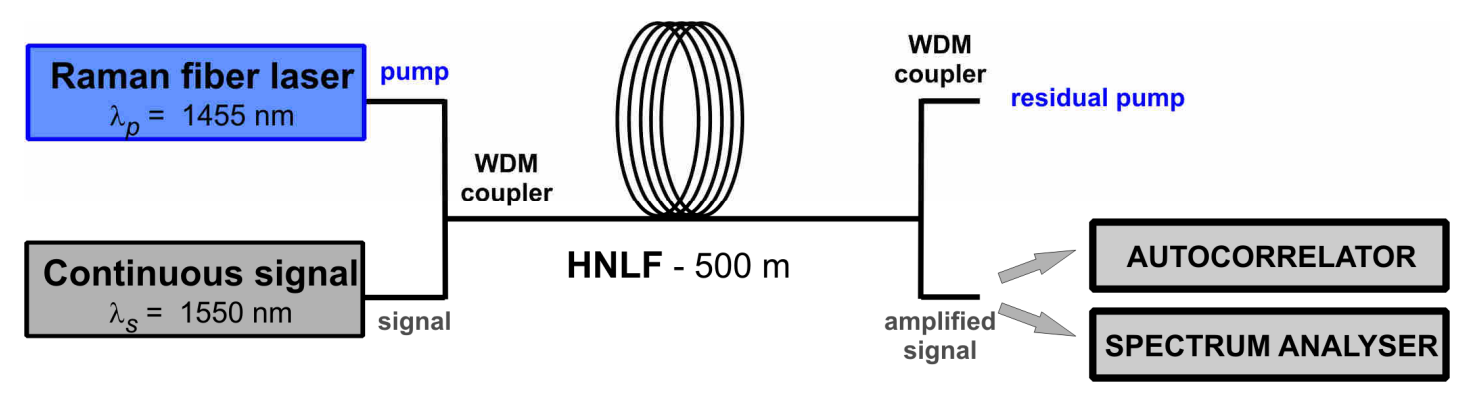

Fig. 8 : Experimental set-up

We have experimentally checked that no significant four-wave mixing between the pump and the signal could be observed, as well as no spontaneous Raman cascading. We may thus consider that the model considered in Eq. 2 is justified. Furthermore, no signature of temporal recompression such as solitonic recompression was observed and the level of spontaneous emission in the amplified signal was maintained very low due to the moderate gain of the considered amplifier.

\subsection{Experimental results}


We have first investigated the impact of the incoherent pumping on the output signal observed in the temporal domain. Results are summarized in Fig. 9. Compared to the pump properties (see Fig. 1(c)), strong change in the contrast of the autocorrelation can be observed, suggesting significant differences in the pulse statistics. Subplots (a) and (b) confirm the strong influence of the walk-off and the depletion effects, which leads in both cases to a temporal broadening of the autocorrelation signal as well as a change in the contrast of the measurement. Indeed, increasing the initial signal level leads to a continuous increase in the autocorrelation function background. Moreover, as regard the influence of the walk-off, we can note that notable changes in the autocorrelation only appear for walk-off values higher than $25 \mathrm{ps}$. These qualitative observations are satisfactory, in that they are fully consistent with the conclusions that have been drawn from our theoretical and numerical analysis. More precisely, systematic and quantitative studies (subplots (c) and (d)) have confirmed this continuous decrease of the contrast ratio combined with an increase of the temporal width, as well as the possible combination of walk-off and pump depletion effects. The quantitative differences compared to the theoretical and numerical results described in the previous sections are mainly ascribed to the use in the experiments regarding the initial level influence of a non-zero walk-off fiber and to polarization issues. The unpolarized nature of the pump wave, as well as the influence of polarization mode dispersion of the fiber can indeed strongly affect the signal evolution $[14,45]$. In spite of its interest, such a study goes beyond the scope of the present article. 

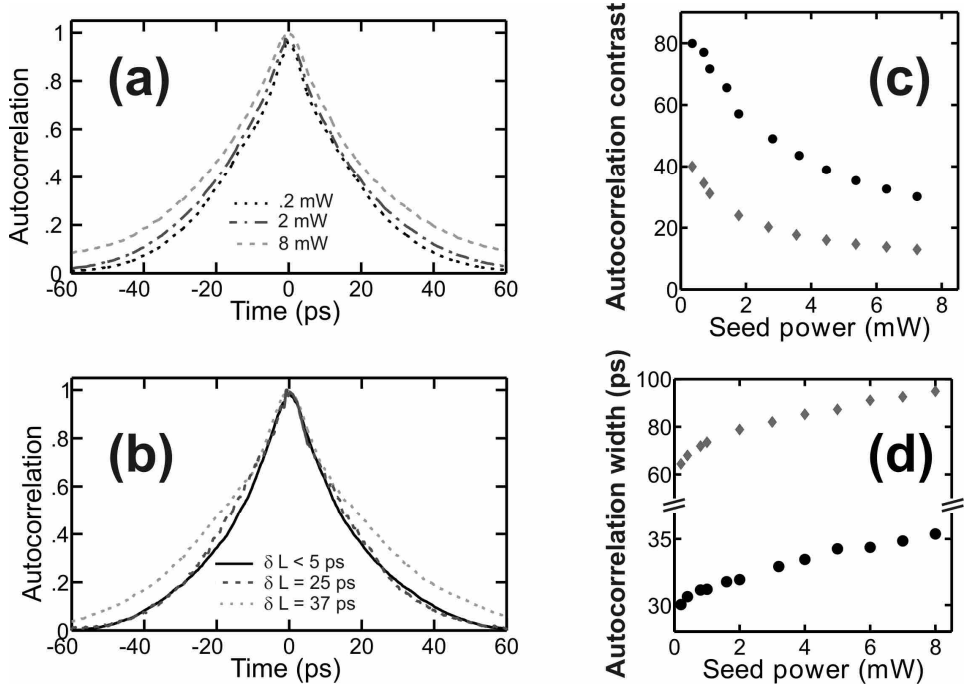

Fig. 9 : Output autocorrelation of the amplified signal intensity: (a) For various initial signal levels (same convention as Fig. 4(b)) (b) For various values of the walk-off between the signal and the pump (0ps, 25 ps and $37 \mathrm{ps,} \mathrm{black} \mathrm{solid,} \mathrm{grey} \mathrm{dashed} \mathrm{and} \mathrm{light} \mathrm{grey} \mathrm{dotted} \mathrm{lines} \mathrm{respectively).} \mathrm{Influence} \mathrm{of} \mathrm{the} \mathrm{initial}$ signal level on the contrast of the autocorrelation (c) and its temporal width (d) for initial walk-off values of $25 \mathrm{ps}$ and $37 \mathrm{ps}$ (black circles and grey diamonds).

Additional measurements have been carried out in the spectral domain. Corresponding results for various levels of initial signal and various amounts of walk-off are summarized on Fig. 10 and again confirm the strong impact of the two parameters. Clearly, a narrowing of the output spectrum is observed when depletion or walk-off increase, which is fully consistent with the results of our theoretical and numerical analysis.
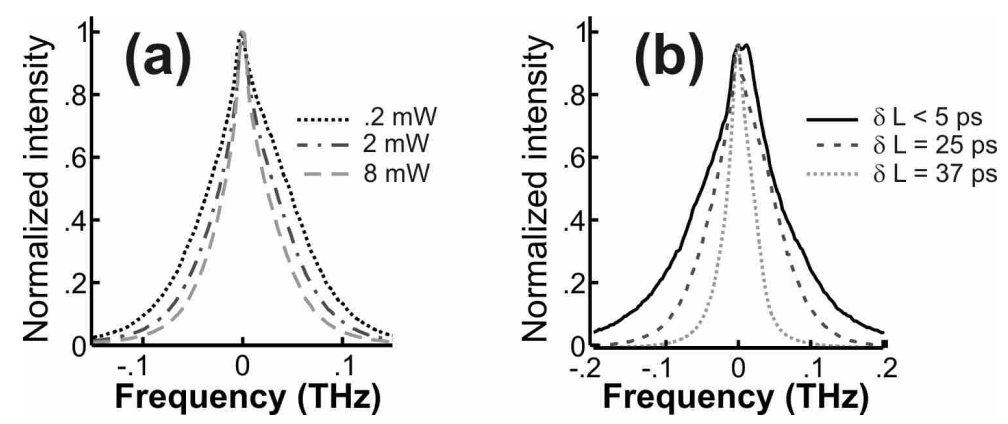

Fig. 10 : Output spectra of the amplified signal : (a) For various initial signal levels (same convention as Fig. 4(b)) (b) For various values of the walk-off between the signal and the pump (same convention as Fig. 9(b)). The resolution of the optical spectrum analyzer is $10 \mathrm{GHz}$. 


\section{Conclusion}

A theoretical, numerical and experimental analysis of the impact of the pump depletion and of the walk-off effects has enabled us to provide some clues for a better understanding of the amplification process taking place in discrete highly nonlinear Raman fiber amplifiers pumped in a copropagating scheme by a partially incoherent wave. Depletion and walk-off can both shorten the pump-signal interaction length, leading to a substantial reshaping of the statistical properties of the output signal. In other words, the generation of the most intense optical peaks is stimulated by negligible depletion effect combined with low walk-off values. On the contrary, reducing output fluctuations requires a high walk-off and/or a saturation of the amplifier. Such conclusions have been derived by an analysis of the probability distribution functions of the instantaneous powers of the waves. The incoherent nature of the pump delivered by a Raman fiber laser has been taken into account in the temporal domain and the various trends are in satisfactory agreement with previous results based on the evaluation of the relative intensity noise evolution during the amplification process [19]. The optical spectrum is also highly affected, experiencing a significant broadening caused by cross phase modulation of the pump on the signal. Such conclusions may find applications in the design of lumped Raman fiber amplifiers [46], especially in the case of wavelength multiplexed signals where the large number of channels under operation leads to rapid pump depletion and where each channel undergoes a different amount of walk-off as well as potential spectral broadening.

Let us finally note that the analytical computations of the pdf reported here may easily be extended to phase-sensitive parametric amplifiers driven from an incoherent pump $[16,17,35$, 47], as well as to multidimensional spatio-temporal optical systems, in which the interplay of the spatial and temporal coherence properties may exhibit intriguing dynamical features [48, 49]. 
The analysis of extreme statistics in phase-sensitive parametric amplifiers is also relevant to plasma physics, in particular as regards the important issue of inertial confinement fusion, in which the coherence properties of parametric instabilities were shown to be essential for the ultimate control of the confinement process (see, e.g., [50]). 


\section{Appendix}

The derivation of the pdf of the signal intensity (25) proceeds in several steps. We recall that we consider here the influence of the mutual walk-off between the two waves by neglecting the depletion of the pump wave. First of all we consider the evolution of the signal intensity in its own reference frame, in which it sees a pump that fluctuates with an effective correlation length $\varepsilon$ $=\tau_{c} / \delta$, where $\tau_{\mathrm{c}}$ is the coherence time of the pump fluctuations. Because we neglect pump depletion, in this reference frame the evolution of the signal at some given time $t_{0}$ is decoupled from its evolution at a neighboring time $t$, so that the equation for the signal intensity $P_{S}$ reduces to an ordinary differential equation. The corresponding solution reads $P_{S}(z)=P_{S 0} \exp \left[\int_{0}^{z} \widetilde{P}_{P}(x) d x\right]$, where $\tilde{P}_{P}(x)$ denotes the pump intensity random function characterized by the effective correlation length $\varepsilon$. Note that, for convenience, we normalized the problem with respect to the amplification length $L_{\mathrm{amp}}=1 /\left(\mathrm{g}_{\mathrm{R}}\left\langle P_{P}\right\rangle\right),\left\langle P_{P}\right\rangle$ being the average pump intensity. The variables can be recovered in real units through the transformation: $z \rightarrow z$ $L_{\text {amp }} ; P_{S, P} \rightarrow P_{S, P}\left\langle P_{P}\right\rangle$. Within these units we have $\operatorname{pdf}\left(\tilde{P}_{P}\right)=H\left(\tilde{P}_{P}\right) \exp \left(-\tilde{P}_{P}\right)$, with $\left\langle\tilde{P}_{P}\right\rangle=1$ and $\left\langle\tilde{P}_{P}^{2}\right\rangle=2$.

We shall begin to compute the pdf of the random function $y(z)=\int_{0}^{z} \widetilde{P}_{P}(x) d x$. For this purpose we write the integral into the following discrete sum, $y(z)=z+\int_{0}^{z} \eta(x) d x \cong z\left(1+R_{N}\right)$, where $R_{N}=\frac{1}{N} \sum_{i=1}^{N} \eta_{i}, \eta(x)=\tilde{P}_{P}(x)-1$, and $z=N \varepsilon$. With this discretization, the sampling of the 
random function $\eta(x)$ 'involves uncorrelated random variables' $\eta_{i}$, which allows us to assume that the random variables $\eta_{i}$ are independent from each other. We shall now calculate the pdf of the random variable $R_{N}=\frac{1}{N} \sum_{i=1}^{N} \eta_{i}$, recalling that the pdf of $\eta_{i}$ is deduced from the pdf of $\tilde{P}_{P}$. It reads $\operatorname{pdf}\left(\eta_{i}\right)=H\left(\eta_{i}+1\right) \exp \left(-\left(\eta_{i}+1\right)\right)$, with $\left\langle\eta_{i}\right\rangle=0$ and $\left\langle\eta_{i}^{2}\right\rangle=1$. Because the random variables $\eta_{i}$ are independent, the pdf of $R_{N}$ is simply given by the product of $N$ convolutions of $p d f\left(\eta_{i}\right)$ [22]. Such a convolution may easily be calculated in the Fourier domain by means of the corresponding characteristic functions [22]. We obtain

$$
M_{R_{N}}(\omega)=\left\langle\exp \left(i \omega R_{N}\right)\right\rangle=\left[M_{\eta_{i}}(\omega)\right]^{N}=\frac{\exp (-i N \omega)}{(1-i \omega)^{N}}
$$

Making use of the residue theorem, the inverse Fourier transform gives the pdf of $R_{N}$

$$
p d f\left(R_{N}\right)=H(R+1) \frac{N^{N}}{(N-1) !}(R+1)^{N-1} \exp [-N(R+1)]
$$

where the symbol '!' denotes the factorial operation. We underline that this expression differs substantially from the expression obtained applying the Central Limit Theorem (CLT). Indeed, because the random variables $\eta_{i}$ are independent, the pdf of their sum $R_{N}$ is provided by the CLT in the limit of large $N$, which readily gives the following Gaussian distribution

$$
p d f^{C L T}\left(R_{N}\right)=\sqrt{\frac{N}{2 \pi}} \exp \left(-\frac{N R^{2}}{2}\right),
$$

with the corresponding variance $\sigma=1 / \sqrt{N}$. A comparative plot of Eq. (A2) and (A3) reveals that the two distributions differ significantly: In contrast with $p d f\left(R_{N}\right)$, the distribution $p d f^{C L T}\left(R_{N}\right)$ does not exhibit a long tail, as illustrated in Fig. A1 for a relatively large value of $N$. It is only for very large values of $N$, i.e. $N>10^{3}$, that Eq.(A3) provides a good approximation 
of Eq.(A2). We also note that in the limit $N \rightarrow \infty$, the variance of the Gaussian distribution (A3) tends to zero $(\sigma \rightarrow 0)$, and the pdf (A2) and (A3) both tend to the Dirac distribution, $p d f^{C L T}\left(R_{N}\right)=\delta\left(R_{N}\right)$. This is consistent with the intuitive idea that in the limit of a very small effective correlation length $\varepsilon$ (i.e. large walk-off $\delta$, or small correlation time $\tau_{c}$ ), the fluctuations of the pump are averaged out and the signal effectively sees a fully coherent (deterministic) pump wave. Finally, to calculate the pdf of the signal intensity $P_{S}$, one may proceed according to standard rules of pdf transformations [31]. Using the pdf of $R_{N}$ given in Eq.(A2), we obtain

$$
p d f\left(P_{S}\right)=\frac{H\left(P_{S}-P_{S 0}\right)}{\varepsilon^{z / \varepsilon} P_{S}\left(\frac{z}{\varepsilon}-1\right) !}\left(\frac{P_{S 0}}{P_{S}}\right)^{1 / \varepsilon}\left[\ln \left(\frac{P_{S}}{P_{S 0}}\right)\right]^{\frac{z}{\varepsilon}-1},
$$

which precisely corresponds to the pdf (25) in normalized units. We remark that in the limit of a small effective correlation length $\varepsilon$ (i.e., large $\delta$ or small $\tau_{c}$ ), the pdf of the signal intensity (A4) takes the simple form $p d f\left(P_{S}\right)=\delta\left[P_{S}-P_{S 0} \exp (z)\right]$, where $\delta$ denotes here the Dirac $\delta$-function: the signal gets amplified as in the fully coherent case. This may be shown by recalling that in the limit $\varepsilon \ll 1$, the CLT gives $p d f\left(R_{N}\right)=\delta\left(R_{N}\right)$. Applying again the standard rules of pdf transformations [22], one readily obtains $p d f\left(P_{S}\right)=\delta\left[P_{S}-P_{S 0} \exp (z)\right]$.
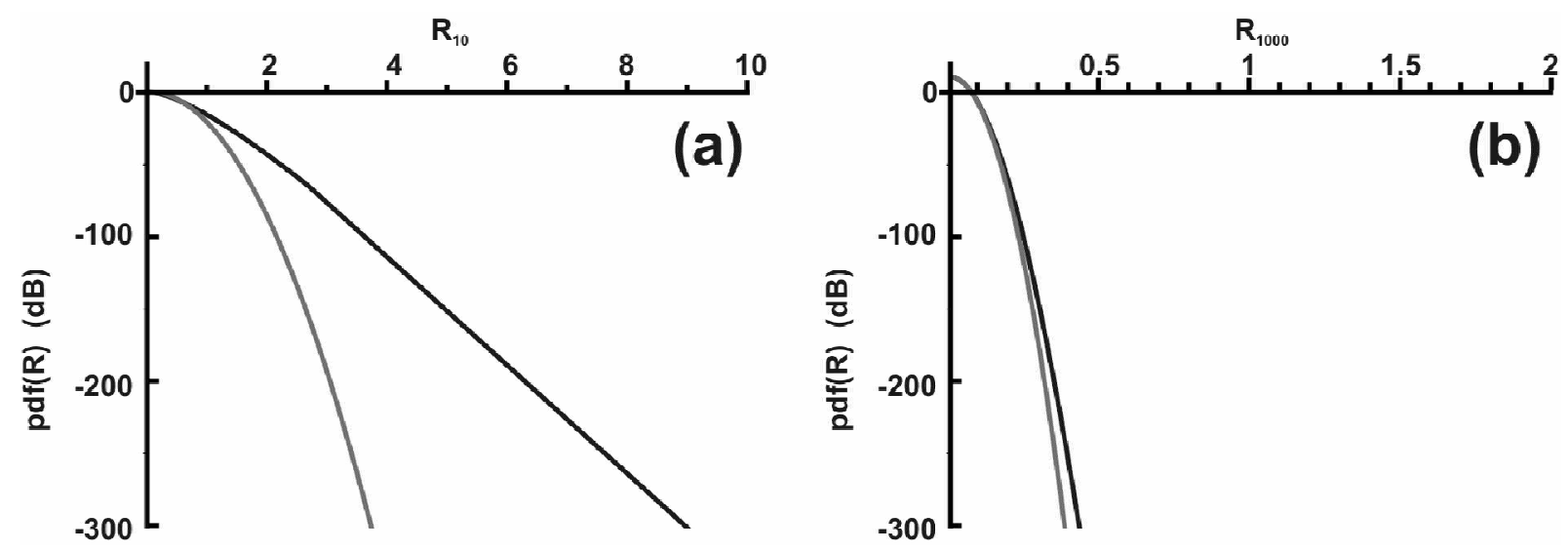
Fig. A1 : Pdf of the random variable $\mathrm{R}_{\mathrm{N}}$ obtained from Eq. (A2) (black solid line) and Eq. (A3) (grey solid line) for (a) $\mathrm{N}=10$ and (b) $\mathrm{N}=1000$.

\section{Acknowledgments}

We would like to thank Guy Millot for stimulating discussions. This work was supported by the Agence Nationale de la Recherche (ANR MANUREVA, PERSYST II and COSTUME projects: ANR-08-SYSC-019, ANR-07-TCOM-014 and ANR-08-SYSC-004-03) and by the Conseil

Régional de Bourgogne. The experimental work has benefited from the PICASSO Platform equipment.

\section{References}

[1] K. Tai, A. Hasegawa, A. Tomita, Phys. Rev. Lett., 56 (1986) 135.

[2] L.F. Mollenauer, R.H. Stolen, J.P. Gordon, Phys. Rev. Lett., 45 (1980) 1095.

[3] C. Finot, J.M. Dudley, B. Kibler, D.J. Richardson, G. Millot, IEEE J. Quantum Electron., 45 (2009) 1482-1489.

[4] D.R. Solli, C. Ropers, P. Koonath, B. Jalali, Nature, 450 (2007) 1054-U1057.

[5] M. Taki, A. Mussot, A. Kudlinski, E. Louvergneaux, M. Kolobov, M. Douay, Phys. Lett. A, 374 (2010) 691-695.

[6] J.M. Dudley, C. Finot, G. Millot, J. Garnier, G. Genty, D. Agafontsev, F. Dias, Eur. Phys. J.Spec. Top., 185 (2010) 125-133.

[7] A. Aalto, G. Genty, J. Toivonen, Opt. Express, 18 (2010) 1234-1239.

[8] E.G. Turitsyna, G. Falkovich, V.K. Mezentsev, S.K. Turitsyn, Phys. Rev. A, 80 (2009).

[9] A. Peleg, Opt. Lett. , 29 (2004) 1980-1982.

[10] Y.J. Chung, A. Peleg, Nonlinearity, 18 (2005) 1555-1574.

[11] K. Hammani, C. Finot, J.M. Dudley, G. Millot, Opt. Express, 16 (2008) 16467-16474. 
[12] C. Finot, K. Hammani, J. Fatome, J.M. Dudley, G. Millot, IEEE J. Quantum Electron., 46 (2010) 205-213.

[13] D. Borlaug, S. Fathpour, B. Jalali, IEEE Photonics J., 1 (2009) 33-39.

[14] C. Headley, G.P. Agrawal, Raman Amplification in Fiber Optical Communication Systems, Academic Press, 2005.

[15] J. Bromage, J. Lightwave Technol., 22 (2004) 79-93.

[16] K. Hammani, C. Finot, G. Millot, Opt. Lett., 34 (2009) 1138-1140.

[17] Y.Q. Xu, S.G. Murdoch, Opt. Lett. , 35 (2010) 826-828.

[18] K. Hammani, C. Finot, B. Kibler, G. Millot, IEEE Photonics J., 1 (2009) 205-212.

[19] C.R.S. Fludger, V. Handerek, R.J. Mears, J. Lightwave Technol., 19 (2001) 1140-1148.

[20] M. Krause, S. Cierullies, H. Renner, E. Brinkmeyer, Opt. Commun., 260 (2006) 656-661.

[21] K. Keita, P. Delaye, R. Frey, G. Roosen, J. Opt. Soc. Am. B: Opt. Phys., 23 (2006) 24792485.

[22] M.D. Mermelstein, C. Headley, J.C. Bouteiller, Electron. Lett., 38 (2002) 403-405.

[23] B. Bristiel, S.F. Jiang, P. Gallion, E. Pincemin, IEEE Photonics Technol. Lett., 18 (2006) 980-982.

[24] G.P. Agrawal, Nonlinear Fiber Optics, Fourth Ed., Academic Press, San Francisco, CA, 2006.

[25] D. Dahan, G. Eisenstein, Opt. Commun., 236 (2004) 279-288.

[26] C. Headley, G.P. Agrawal, IEEE J. Quantum Electron., 31 (1995) 2058-2067.

[27] F. Vanholsbeeck, S. Martin-Lopez, M. Gonzalez-Herraez, S. Coen, Opt. Express, 13 (2005) 6615-6625.

[28] B. Barviau, S. Randoux, P. Suret, Opt. Lett. , 31 (2006) 1696-1698.

[29] J. Schroder, S. Coen, Opt. Express, 17 (2009) 16444-16449.

[30] Note that the Fourier transform is considered here in the sense of the distributions, as it is usual in wave turbulence theory, see e.g., V.E. Zakharov, V.S. L'vov, G. Falkovich, Kolmogorov Spectra of Turbulence I Springer, Berlin, 1992. 
[31] J.W. Goodman, Statistical Optics, John Wileay and Sons, 1985.

[32] A. Betlej, P. Schmitt, P. Sidereas, R. Tracy, C.G. Goedde, J.R. Thompson, Opt. Express, 13 (2005) 2948-2960.

[33] E. Landahl, D. Baiocchi, J.R. Thompson, Opt. Commun., 150 (1998) 339-347.

[34] J. Chang, D. Baiocchi, J. Vas, J.R. Thompson, Opt. Commun., 139 (1997) 227-231.

[35] Y.Q. Xu, S.G. Murdoch, Opt. Lett. , 35 (2010) 169-171.

[36] L. Garcia, A. Jalili, Y. Lee, N. Poole, K. Salit, P. Sidereas, C.G. Goedde, J.R. Thompson, Opt. Commun., 193 (2001) 289-300.

[37] Y.J. Chen, A.W. Snyder, J. Lightwave Technol., 7 (1989) 1109-1117.

[38] G. Ravet, A.A. Fotiadi, P. Megret, in CLEO Europe 2007, 2007.

[39] B.E. Olsson, P. Ohlen, L. Rau, D.J. Blumenthal, IEEE Photonics Technol. Lett., 12 (2000) 846-848.

[40] R. Holzlohner, C.R. Menyuk, Opt. Lett. , 28 (2003) 1894-1896.

[41] A.S. Grabtchikov, A.I. Vodtchits, V.A. Orlovich, Phys. Rev. A, 56 (1997) 1666-1669.

[42] G.P. Agrawal, P.L. Baldeck, R.R. Alfano, Phys. Rev. A, 40 (1989) 5063.

[43] C. Finot, Opt. Commun., 249 (2005) 553-561.

[44] C.H. Kwok, C. Lin, IEEE J. Sel. Top. Quantum Electron., 12 (2006) 451-458.

[45] C. Martinelli, L. Lorcy, A. Durecu-Legrand, D. Mongardien, S. Borne, J. Lightwave Technol., 24 (2006) 3490-3505.

[46] T. Miyamoto, M. Tanaka, J. Kobayashi, T. Tsuzaki, M. Hirano, T. Okuno, M. Kakui, M. Shigematsu, J. Lightwave Technol., 23 (2005) 3475-3483.

[47] A. Picozzi, C. Montes, M. Haelterman, Phys. Rev. E, 66 (2002) 056605.

[48] A. Picozzi, M. Haelterman, Phys. Rev. Lett., 88 (2002) 083901.

[49] O. Jedrkiewicz, A. Picozzi, M. Clerici, D. Faccio, P. Di Trapani, Phys. Rev. Lett., 97 (2006) 243903.

[50] J. Fuchs, C. Labaune, H. Bandulet, P. Michel, S. Depierreux, H.A. Baldis, Phys. Rev. Lett., 88 (2002) 195003. 
